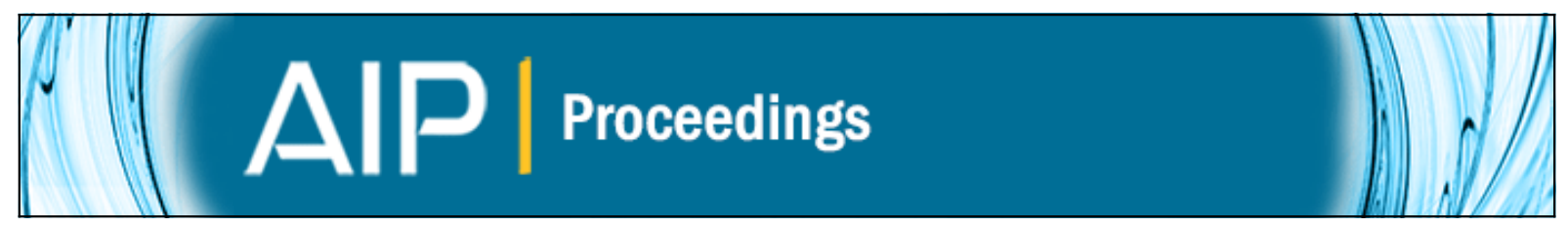

\title{
Dry particle coating of polymer particles for tailor-made product properties
}

C. Blümel, J. Schmidt, A. Dielesen, M. Sachs, B. Winzer, W. Peukert, and K.-E. Wirth

Citation: AIP Conference Proceedings 1593, 248 (2014); doi: 10.1063/1.4873774

View online: http://dx.doi.org/10.1063/1.4873774

View Table of Contents: http://scitation.aip.org/content/aip/proceeding/aipcp/1593?ver=pdfcov

Published by the AIP Publishing

Articles you may be interested in

Complete tailor-made inverse filter for image processing of scanning SQUID microscope

Appl. Phys. Lett. 100, 182601 (2012); 10.1063/1.4709492

Finite Element Modeling of Transition Zone in Friction Stir Welded TailorMade Blanks

AIP Conf. Proc. 908, 1457 (2007); 10.1063/1.2741014

Microwave photonic crystal with tailor-made negative refractive index

Appl. Phys. Lett. 85, 1858 (2004); 10.1063/1.1787959

Photon scanning tunneling microscopy of tailor-made photonic structures

Appl. Phys. Lett. 77, 142 (2000); 10.1063/1.126903

Tailor-made quantum states

Phys. Today 


\title{
Dry Particle Coating of Polymer Particles for tailor-made Product Properties
}

\author{
C. Blümel, J. Schmidt, A. Dielesen, M. Sachs, B. Winzer, W. Peukert, K.-E. Wirth* \\ Institute of Particle Technology; University of Erlangen- Nuremberg; Germany \\ www.lfg.uni-erlangen.de; *karl-ernst.wirth@fau.de
}

\begin{abstract}
Disperse polymer powders with tailor-made particle properties are of increasing interest in industrial applications such as Selective Laser Beam Melting processes (SLM). This study focuses on dry particle coating processes to improve the conductivity of the insulating polymer powder in order to assemble conductive devices. Therefore PP particles were coated with Carbon Black nanoparticles in a dry particle coating process. This process was investigated in dependence of process time and mass fraction of Carbon Black. The conductivity of the functionalized powders was measured by impedance spectroscopy. It was found that there is a dependence of process time, respectively coating ratio and conductivity. The powder shows higher conductivities with increasing number of guest particles per host particle surface area, i.e. there is a correlation between surface functionalization density and conductivity.

The assembled composite particles open new possibilities for processing distinct polymers such as PP in SLM process. The fundamentals of the dry particle coating process of PP host particles with Carbon Black guest particles as well as the influence on the electrical conductivity will be discussed.
\end{abstract}

Keywords: dry particle coating, agglomeration, impedance spectroscopy, conductivity, percolation

\section{INTRODUCTION}

Whether cars, planes, implants, or sport equipments almost every industry sector is interested in tailor-made light weight constructions. SLM processes can provide both: devices made from polymers as well as tailormade designs due to formless manufacturing. To come up to these continuously rising requirements fine polymer powders with adjustable particle properties are necessary. Dry particle coating is an economic and easy-handling mixing process for assembling tailormade composite polymer particles (FIGURE 1).

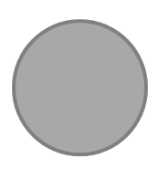

host particle

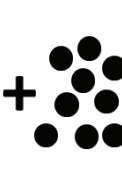

guest particles

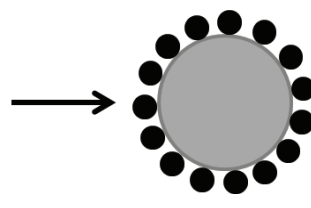

composite particle
FIGURE 1: dry particle coating

Therefore nanoscale guest particles (FIGURE 1, middle) are attached to the surface of microscale host particles (FIGURE 1, left) mainly by van-der-Waals forces in order to obtain composite particles (FIGURE 1, right). Dry particle coating processes have already been conducted with several host particles pursuing different aims, such as increase of flowability $[1 ; 2 ; 3]$, reduction of wettability [4] or improvement of electrochemical performance of graphite anodes in lithium ion batteries [5].
For applications that demand conductive devices that are to be built via SLM, the basic raw material, i.e. the powder itself must exhibit an appropriate conductivity. One option to increase the electrical conductivity of originally insulating matter like the most common polymers is the attachment of 'guest' material showing good conductivity at the surface of the host particles: at sufficiently high surface functionalization density, percolation will occur and the powder packing will exhibit a remarkably increased conductivity.

To the authors' knowledge so far there is nothing reported concerning dry particle coating of polymer particles for SLM processing in order to increase conductivity of polymer powders.

\section{EXPERIMENTAL}

\section{Dry particle coating}

Polypropylene (PP) particles, used in the following as host particles, were coated with Carbon Black guest particles. Table 1 summarizes selected properties of the used host and guest particles.

TABLE 1: properties of host particles and guest particles

\begin{tabular}{|c|c|c|c|c|}
\hline & & producer & $\mathrm{x}_{50,3}$ & $\begin{array}{c}\text { density } \\
/ \mathrm{kg} \mathrm{m}^{-3}\end{array}$ \\
\hline $\begin{array}{c}\text { host } \\
\text { particles }\end{array}$ & $\begin{array}{c}\text { polypropylene } \\
\text { (Coathylene } \\
\text { PD0580) }\end{array}$ & DuPont & $55 \mu \mathrm{m}$ & 910 \\
\hline $\begin{array}{c}\text { guest } \\
\text { particles }\end{array}$ & $\begin{array}{c}\text { Carbon Black } \\
\text { (Super P) }\end{array}$ & Timcal & $40 \mathrm{~nm}$ & 2200 \\
\hline
\end{tabular}

The dry particle coating processes were conducted using a tumbling mixer (T2F, Willy A. Bachofen AG). The PP host particles were mixed at a rotation speed of 
49 rpm with Carbon Black guest particles (0.05$5.0 \mathrm{wt} \%$ ) in $600 \mathrm{ml}$ aluminum bottles for varying process times.

Taking samples at different process times enable us to find the optimum process time for obtaining a homogeneous distribution of guest particles on the surface of the host particles. Furthermore the different coating phases can be investigated to understand the coating mechanisms.

\section{Characterization of the coating}

The quality of the coating has been characterized by scanning electron microscopy (Zeiss, Gemini Ultra 55; InLens detector). Pictures with different magnifications were taken from the uncoated particles as well as from the composite particles. For quantitative analysis of the coating, the number of agglomerates per host particle surface area was determined using the SEM images with the highest magnification. Therefore several SEM images were divided in squares with $1 \mu \mathrm{m}$ edge length and the number of agglomerates within this area was counted, see FIGURE 2.

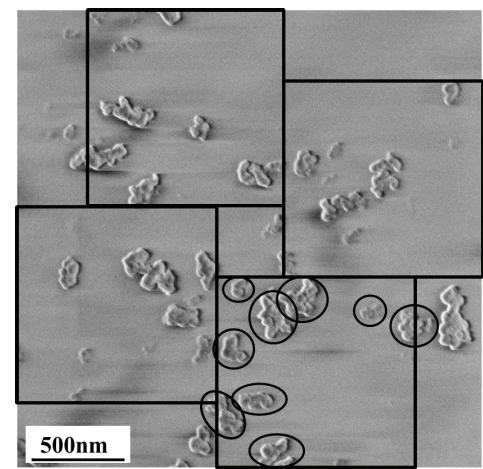

FIGURE 2: quantitative analysis: number of agglomerates

\section{Impedance Spectroscopy}

For characterization of the conductivity behavior of the powders, impedance measurements have been performed using the dielectric spectrometer AlphaAnalyzer (Novocontrol). In order to get reproducible results the packing density of the powders has to be adjusted by a reproducible procedure. Therefore pellets of the composite particles of defined geometry have been prepared at constant load conditions: a pellet compacter (Zwick Z020) has been operated at a load of $20 \mathrm{kN}$. The round pellets show a diameter of $20 \mathrm{~mm}$ and - depending on the powder characteristics and mass applied - a height of $30 \mathrm{~mm}$ to $60 \mathrm{~mm}$.

The dielectric measurements were performed at a constant temperature of $20^{\circ} \mathrm{C}$ in the frequency range $10^{-4} \mathrm{~Hz}$ to $20 \mathrm{MHz}$ (at $1 \mathrm{~V}$ (root mean square)) in nitrogen atmosphere. Before performing the measurements the sample cell has been thoroughly flushed with nitrogen and has been equilibrated at the destined temperature for $15 \mathrm{~min}$ which was sufficient to attain thermal equilibrium. The temperature has been maintained constant during impedance characterization with accuracy of $0.1 \mathrm{~K}$ by purging with dry nitrogen gas flow of desired temperature using the Quatro Cryosystem (Novocontrol). Impedance data have been evaluated using WinFit software (Novocontrol).

\section{RESULTS AND DISCUSSION}

\section{Dry Particle Coating}

The uncoated PP host particle surface is displayed in FIGURE 3 (left). A homogeneous coated host particle is obtained after dry particle coating processes with $0.5 \mathrm{wt} \%$ Carbon Black guest particles shown in FIGURE 3 (right).
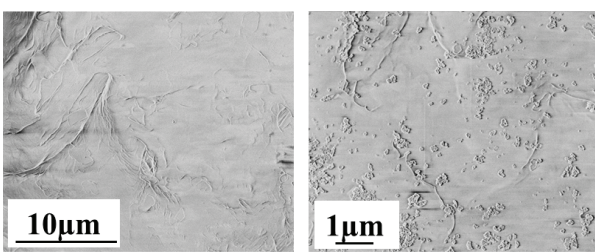

FIGURE 3: PP host particle (left); PP $+0.5 \mathrm{wt} \%$ Carbon Black composite particle (right)

FIGURE 4 (top) shows 2 pressed pellets, both containing PP and $0.5 \mathrm{wt} \%$ Carbon Black. While the pellet pressed from powder withdrawn after $120 \mathrm{~min}$ coating time has a homogeneous dye distribution, the pellet pressed from powder withdrawn after 1 min process time shows several great Carbon Black agglomerates (black dots) embedded in the white polymer matrix. This can also be observed looking at the microstructure (FIGURE 4 bottom): In comparison to the process time of $1 \mathrm{~min}$ where almost no coating is obtained, the SEM image after $120 \mathrm{~min}$ process time shows a homogeneous coating of the PP host particle even at the sub-micron scale.

\section{PP + 0.5wt\% Carbon Black}

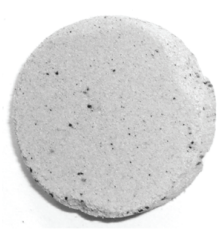

$1 \mathrm{~min}$
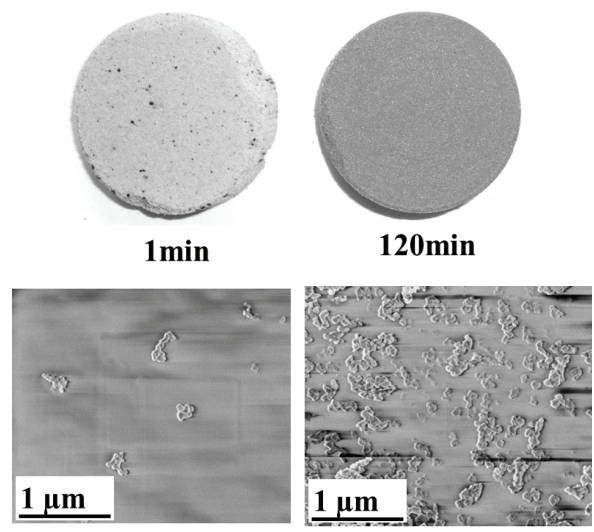

FIGURE 4: PP particles coated with $0.5 \mathrm{wt} \%$ Carbon Black guest particles: top: macroscopic appearance; below: microscopic appearance (left: $1 \mathrm{~min}$, right: 120min)

FIGURE 5 displays the number surface density of agglomerates in dependence of the process time for varying Carbon Black mass fractions. It can be seen that with increasing guest particle concentration the 
number of agglomerates increases and therefore the occupancy rate, see FIGURE 6. There is an optimum distribution of guest particles on the surface of the host particles as a function of process time: longer process times (results are not presented in this paper) lead to mechanofusion processes between guest and host particles. At $5.0 \mathrm{wt} \%$ of Carbon Black a continuous coating of the host particle surface has been observed (FIGURE 6).

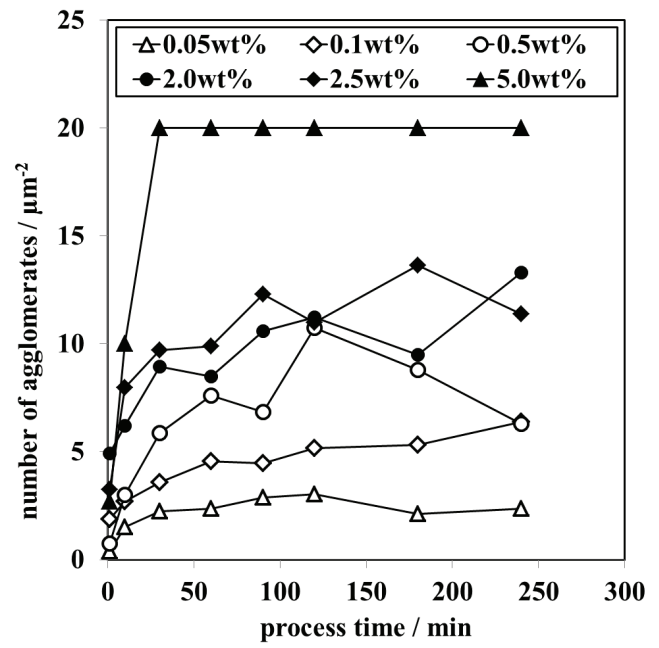

FIGURE 5: number of Carbon Black guest particle agglomerates per host particle unit surface area in dependence of process time. (guest particles: $0.05-5.0 \mathrm{wt} \%$ )

Due to sintering processes via the production of Carbon Black nanoparticles, it can be assumed that these aggregates show an inner porosity of 0.5 (aggregate density: $1100 \mathrm{~kg} \mathrm{~m}^{-3}$ ). Regarding the different densities of host and guest particles, $0.5 \mathrm{wt} \%$ Carbon Black for instance, corresponds to $0.4 \mathrm{vol} \%$ Carbon Black.

A calculation of the maximum agglomerate surface number density indicates that 20 agglomerates per $\mu \mathrm{m}^{2}$ is the highest value that may be observed for Carbon Black guest particles. That is why a value of 20 agglomerates per $\mu \mathrm{m}^{2}$ has been assumed as a limiting value for $5.0 \mathrm{wt} \%$ Carbon Black, though counting is not possible any more.

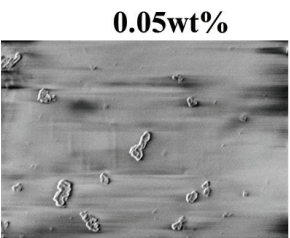

$0.5 w t \%$

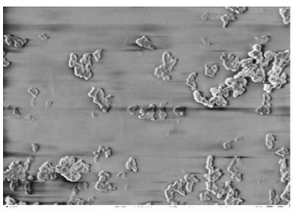

$2.5 w t \%$

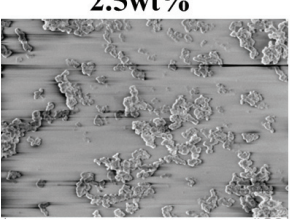

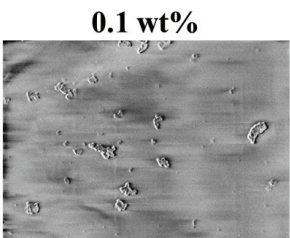

$2.0 \mathrm{wt} \%$

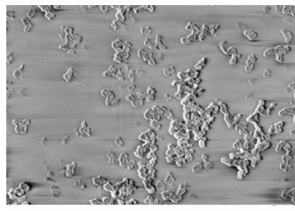

$5.0 w t \%$

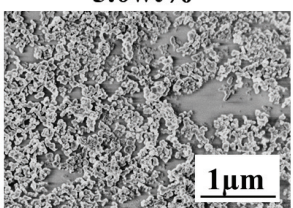

FIGURE 6: SEM images of composite particles with varying guest particle content (30 $\mathrm{min})$

Within FIGURE 7 it becomes apparent that the time to reach the maximum agglomerate surface number density increases with increasing guest particle content until reaching a process time maximum. Adding more guest particles to the coating process leads to a reduction of the process time to reach the maximum number of agglomerates and simultaneously to an increased coating level. It can be stated that with higher guest particle content the saturation of the host particle surface takes place quite earlier than with guest particle contents around $1.0 \mathrm{wt} \%$ regarding this case.

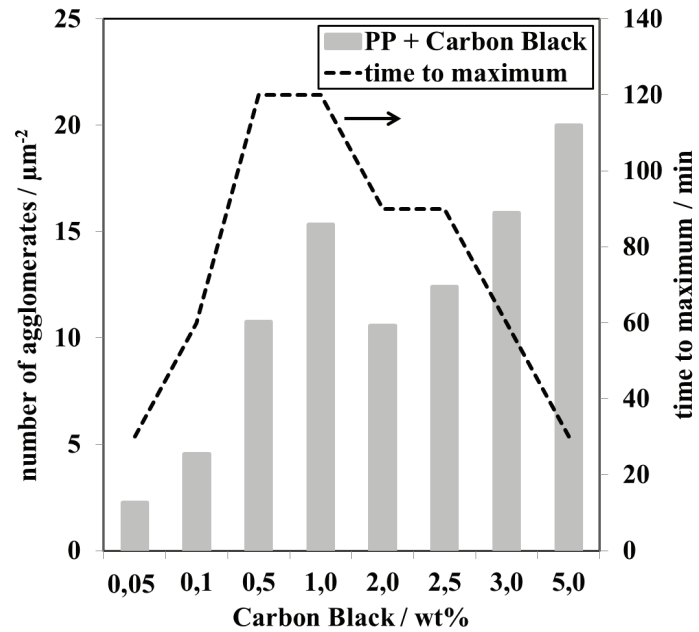

FIGURE 7: maximum number of guest particle agglomerates and time to reach maximum in dependence of guest particle concentration.

Impedance Spectroscopy

The impedance spectroscopic measurements of PP coated with $2.0 \mathrm{wt} \%$ Carbon Black for $120 \mathrm{~min}$ are depicted in FIGURE 8: conductivity and angular phase shift are plotted in dependence on frequency. 


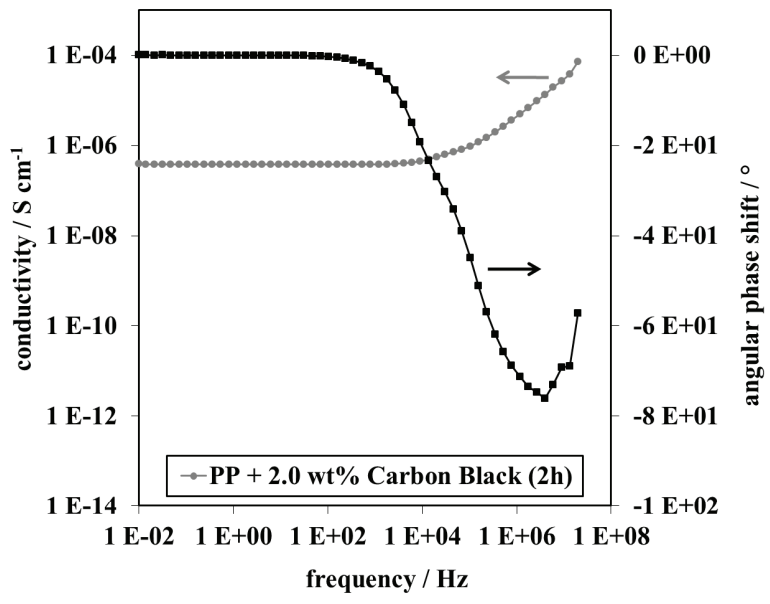

FIGURE 8: PP $+2.0 \mathrm{wt} \%$ Carbon Black: impedance spectroscopy results

It becomes obvious that the composite particles show Ohmic behavior which is characterized by a constant conductivity over a wide frequency range, i.e. the DC plateau for frequencies from approx. $1 \mathrm{MHz}$ to $10^{-2} \mathrm{~Hz}$. Moreover in the aforementioned frequency range the imaginary part of the impedance, i.e. the loss contribution, is virtually zero.

In contrast to the composite particles of $\mathrm{PP}$ and $2.0 \mathrm{wt} \%$ respectively $2.5 \mathrm{wt} \%$ Carbon Black, the composite particle of $\mathrm{PP}$ and $1.0 \mathrm{wt} \%$ Carbon Black show isolating properties, see FIGURE 9. The impedance behavior to a good approximation may be described by a $\left(\omega^{*} \mathrm{C}\right)^{-1}$ dependence (C: capacity) which is typical for insulating materials. The dielectric behavior observed for the composite powder with $1 \mathrm{wt} \%$ Carbon Black is not remarkably different from the non-functionalized polymer.

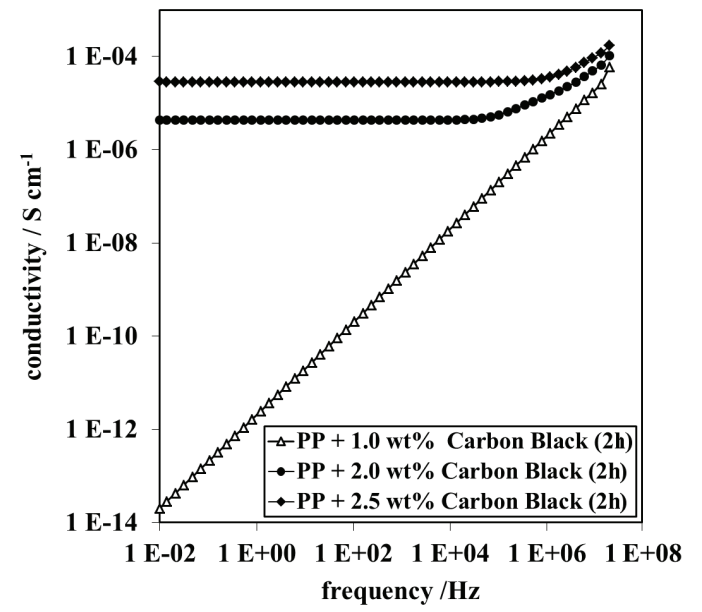

FIGURE 9: $\mathrm{PP}+1.0 \mathrm{wt} \% ; 2.0 \mathrm{wt} \%$ respectively $2.5 \mathrm{wt} \%$ Carbon Black: impedance spectroscopy results

The transition between 'insulating' and 'conductive' composite powder with respect to guest particle concentration is quite sharp. Obviously a critical agglomerate surface functionalization density must be exceeded. This behavior is typical for systems showing percolation.

FIGURE 10 displays the number of guest particle agglomerates and the conductivity of PP coated with $2.0 \mathrm{wt} \%$ Carbon Black in dependence of process time. After $120 \mathrm{~min}$ coating time, the composite material becomes conductive. The number of agglomerates increases with increasing process time.

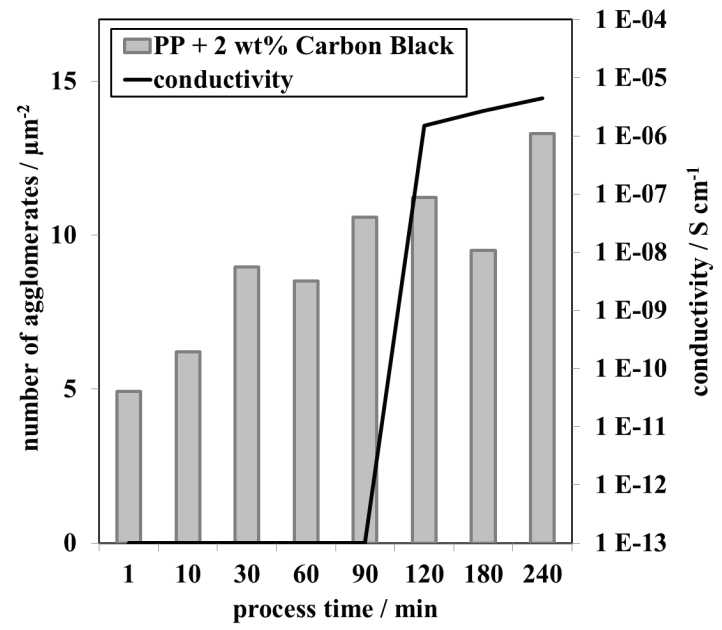

FIGURE 10: number of guest particle agglomerates and conductivity of the composite material in dependence of process time

A more pronounced trend can be observed in FIGURE 11 where the number of guest particle agglomerates and the conductivity of the composite material are shown in dependence of the guest particle content, all at the same process time of $120 \mathrm{~min}$. An increasing number density of agglomerates with increasing mass fraction of Carbon Black leads to a conductivity of the composite particles. Again, a sudden increase of conductivity becomes obvious after a critical surface functionalization density with conducting agglomerates (about $2.0-2.5 \mathrm{wt} \%$ carbon black) is exceeded, which is typical for conductivity mechanism via percolation. 


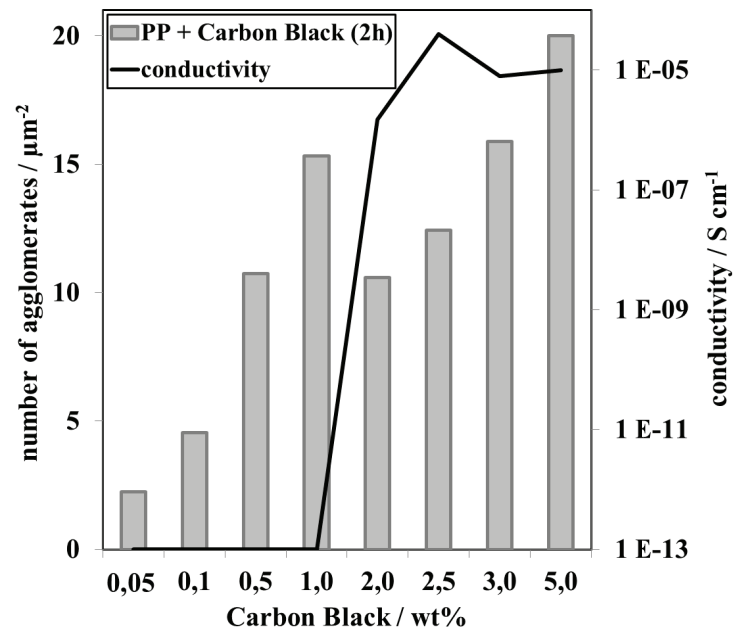

FIGURE 11: number of guest particle agglomerates and conductivity of the composite material in dependence of Carbon Black concentration for constant process time (120 min) and stressing conditions

Selected numbers of guest particles in dependence of the process time are shown in FIGURE 12.

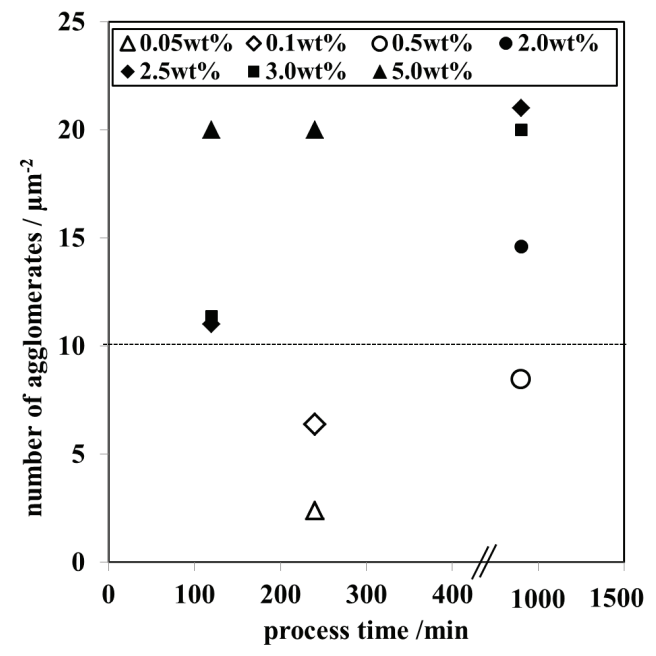

FIGURE 12: number of guest particle agglomerates in dependence of process time: filled symbols: conductive powder; unfilled symbols: no powder conductivity
The filled symbols stand for conductive powder, whereas the unfilled symbols represent the isolating powder. It becomes apparent that about 10 guest particle agglomerates per squares with $1 \mu \mathrm{m}$ edge length are necessary for the conductivity of the investigated PP host particles

\section{CONCLUSIONS}

Dry particle coating processes were conducted to improve the conductivity of polymer powders in order to assemble conductive devices. Therefore PP host particles were coated with Carbon Black guest particles. There is a dependence of process time respectively coating ratio on conductivity. The powder shows higher conductivities with increasing number of guest particle agglomerates per host particle surface area. Therefore a good deagglomeration of the guest particles is necessary. The assembled composite particles can open new possibilities for processing tailor-made polymer particles (e.g. PP coated with carbon black) in the SLM process.

\section{ACKNOWLEDGEMENTS}

This study has been supported by Deutsche Forschungsgemeinschaft (DFG) within the framework of the collaborative research center SFB 814 "Additive Fertigung" (A2, A3). Financial support is gratefully acknowledged.

\section{REFERENCES}

1. H. Rumpf; Chemie-Ingenieur-Technik 1974, 46, 1-11

2. M. Linsenbühler, K.-E. Wirth; Powder Technology 2005, 158, 3-20

3. K. Meyer, I. Zimmermann; Powder Technology 2004, $139,40-54$

4. G. Lefebvre, L. Galet, A. Chamayou; Powder Technology 2011, 208, 372-377

5. S.F. Lux, T. Placke, C. Engelhardt, K.-E. Wirth, M. Winter; Journal of The Electrochemical Society 2011, 159, A1849-A855 